\title{
The Impact of Smart Grid Technology on Dielectrics and Electrical Insulation
}

\author{
V. M. Catterson \\ Institute for Energy and Environment, \\ University of Strathclyde \\ Glasgow, G1 1XW, UK \\ J. Castellon \\ Institut d'Électronique du Sud-Groupe Énergie et Matériaux, \\ Université de Montpellier 2 - CC 079 \\ 34095 Montpellier Cedex 5 - France \\ J. A. Pilgrim \\ Tony Davies High Voltage Laboratory, University of Southampton, \\ Hampshire, SO17 1BJ, UK \\ T. K. Saha, H. Ma \\ School of Information Technology and Electrical Engineering, \\ The University of Queensland, Australia
}

\section{Vakilian, A. Moradnouri, M. Gholami \\ Electrical Engineering Department, Sharif University of Technology, \\ Tehran 11365, Iran}

and B. D. Sparling

Dynamic Ratings Inc.,

Vancouver, BC, Canada

\begin{abstract}
Delivery of the Smart Grid is a topic of considerable interest within the power industry in general, and the IEEE specifically. This paper presents the smart grid landscape as seen by the IEEE Dielectrics and Electrical Insulation Society (DEIS) Technical Committee on Smart Grids. We define the various facets of smart grid technology, and present an examination of the impacts on dielectrics within power assets. Based on the trajectory of current research in the field, we identify the implications for asset owners and operators at both the device level and the systems level. The paper concludes by identifying areas of dielectrics and insulation research required to fully realize the smart grid concept. The work of the DEIS is fundamental to achieving the goals of a more active, self-managing grid.
\end{abstract}

Index Terms — Smart Grids.

\section{INTRODUCTION}

AROUND the world, much effort is focused on transforming power networks from passive, unidirectional, traditional systems to the flexible, self-managing, multi-participant smart grid. Many bodies have published white papers and roadmap documents about how this transition can take place [1-5]. What is often overlooked is the impact of new devices and changing loads on dielectrics and electrical insulation. This paper surveys current trends in smart grid development, and draws out the implications for insulation design and management.

The concept of the smart grid is multi-faceted, and includes different technologies depending on regional drivers. Broadly, the aim is to allow greater demand participation, more distributed energy resources, improved asset usage through dynamic ratings and active network management, and increased reliability and efficiency. These goals can sometimes conflict, and require a significant change to the way networks 
are traditionally operated.

There are two key routes to adopting smart grid technology. To meet the changing objectives, either existing assets are managed and operated differently, or new devices and technologies are deployed. Both cases impact insulation systems. New technologies such as highvoltage direct current (HVDC) place very different demands on insulation, and require a new suite of models and tools for analysis. The alternative-changing the pattern of usage of existing assets - means that existing models of life consumption and fault evolution can no longer be assumed to apply.

The aim of this paper is to bridge between the smart grid and insulation communities. While the paper contains review material of various research topics, a key contribution is in reporting a survey of the landscape of research pertinent to the DEIS technical committee on smart grids. We have examined the various technologies considered under the umbrella of the smart grid, and undertaken a study of how these technologies can impact on electrical insulation. As a result, we foresight their implications for asset owners and operators as various parts of the smart grid come online, both at the materials or device level and at the systems level. Finally, we assess the common themes of smart grid technologies, and draw out the necessary research still required within the dielectrics community before these goals can be achieved.

\section{SMART GRID TECHNOLOGY}

The terminology and focus of smart grid technology varies around the globe, due to the particular challenges and existing grid infrastructure in each region. For example, in North America particular emphasis is placed on advanced metering infrastructure (AMI), demand side participation, electric vehicles, and other load-focused efforts [4], while European smart grids focus on network initiatives, such as dynamic asset ratings, HVDC, and cyber security [5]. China aims to meet rapid load growth with clean generation and improved efficiency [6], while the focus in many parts of Africa is to build the smart grid without legacy transmission grid infrastructure [3].

Despite these differences, there is broad agreement that future electricity networks will strive to incorporate certain concepts, such as:

- Higher reliability through automated fault location and restoration (self-healing),

- Flexible topology and bidirectional flows, making distribution networks more like transmission networks,

- Radically changed load profiles, due to demand side management, embedded generation, and electrification of transport,

- Increased generation from intermittent renewable sources, such as wind and solar,

- Novel markets and services, e.g. power quality and frequency response,

- More communications, leading to more data and an increased need for cyber and physical security.

These concepts rely on the implementation of key technologies, including:

- Dynamic ratings for plant

- Active network management

- HVDC and power electronics

- Communications networks (inter- and intrasubstation)

- Phasor measurement units (PMUs) and increased conventional monitoring of both network and assets

- Smart meters / advanced metering infrastructure

- Storage and electric vehicles.

Widespread deployment of new technology such as offshore HVDC grids has obvious relevance to the dielectrics community. But even some of the topics which have little direct impact on insulation systems (such as changing market structure) are part of a general shift in utilization of network assets which can have unforeseen effects. The following sections review areas of on-going research work on insulation systems in the smart grid.

\section{IMPACT ON DIELECTRICS: MATERIALS AND SENSORS}

New methods and technologies for manufacture of materials present the prospect of improved dielectrics for assets and novel sensors for online monitoring. This section presents some of the areas in which smart grid ideas interact with fundamental research into materials for high voltage devices.

\subsection{SELF-HEALING SYSTEMS}

A primary smart grid concept is that of self-healing, where components can detect and take action to rectify incipient problems before they lead to failure. At the network level, this includes automatic reconfiguration or restoration systems to heal from faults and constraint violations. At the asset level it includes self-healing materials, where components of the system heal the damage from inside, to rehabilitate a failing component. However, it should be noted that not all damage can be repaired, the design of the self-healing mechanism cannot cover every type of damage, and the healing mechanism may itself fail [7].

Polymer compounds suitable for high voltage insulation depend equally on their formulation, the base material, the compounding method, and the curing time or pressure. The properties of interest include electrical, mechanical, and thermal performance, as well as hydrophobicity.

Hydrophobicity is the property of a surface to break up water films and create separate droplets. This property increases resistance to formation of conducting tracks that reduce leakage currents, and also aids in removing loose contamination from the insulator surface. Therefore, increased hydrophobicity gives a level of self-healing capability to outdoor insulators.

The hydrophobic behavior of various materials and preparations has been studied, such as silicon based 
hydrophobic coatings [8], the intrinsic improvement of hydrophobicity of epoxy resins through introducing hydrophobicity to the bulk of the material [9], and the effect of various hardeners and fillers for epoxy resin [10].

Mechanical strength can be retained through incorporation of additives into the polymer matrix prior to fabrication [11], such as aromatic epoxy resins into additive packages. Materials regained a high percentage (75-90\%) of their original mechanical properties; however there are some practical and theoretical concerns about this method, such as storage duration and lifetime of the insulator, physical and electrical properties, and mechanical healing (recovery of mechanical properties).

A self-healing process was shown to generate low molecular weight (LMW) components from high molecular weight (HMW) components by splitting $\mathrm{Si}-\mathrm{O}$ bonds if special boundary conditions were fulfilled [12]. However, further test results showed that the surface recovery produced LMW silicone layers which are not continuous, and the thin lattice type layer permits migration of water and salt in both directions [13].

While there is a desire for new insulation materials with beneficial properties [14, 15], a comprehensive understanding of the long-term dielectric performance is yet to be realized. In particular, the interaction between environmental and electrical aging factors is complex and not fully understood [16]. The environmental factors include ultra violet radiation from sunlight, organic growth, acid rain and ozone; and the electrical factors are primarily corona from water droplets or metallic fittings, and dryband arcing.

\subsection{SELF-MANAGING SYSTEMS}

Another aim of the smart grid is that networks become self-managing, for example by increasing automation and monitoring to reduce the number and duration of outages. This requires improved sensors and techniques for monitoring, to gain a full understanding of asset health and network state.

One example is pollution monitoring on insulator strings, to allow flashover to be predicted and avoided. Many approaches require manual inspection or short circuit testing (such as ultraviolet, infrared, or ultrasonic monitoring, the liquid water sensor (LWS), and electrical methods $[17,18])$, and are therefore not suitable for remote, automated implementation.

In comparison, monitoring of the leakage current and pulse current can be performed remotely. Leakage current gives partial information of the operating state of the insulator, while pulse current highlights the presence of corona discharge or partial discharge due to fissures in the insulator. A light emitting diode (LED) can be used to show the leakage current, with advantages like low energy usage, high reliability, long life, and low maintenance. Since the technique utilizes passive components and photoelectric coupling, it is also resilient to noise and well insulated. When leakage current flows, the LED in the sensor head flashes, and the signal is sent through optical fiber to the detector module [19]. Further stages of amplification and filtering can be added [20].

An alternative to these monitoring practices is to analyze acoustic emissions, since the materials have distinctive characteristics of acoustic emission under different stress conditions [21]. This can be used to detect pollution buildup and evaluate contamination severity of insulators.

\subsection{HVDC}

Future power networks are likely to be characterized by longer transmission distances, to connect remote generation such as off-shore wind farms and large scale hydro generation; and to stabilize national networks through interconnection. For long distance energy transmission, direct current has been preferred to alternating current. The development of High Voltage Direct Current (HVDC) cables requires design according to specific criteria and materials with appropriate properties.

Cross-linked polyethylene (XLPE) has established itself over the past 20 years as the most used insulation material for HVAC cables, but also more recently for HVDC cables. Among the different cable technologies used for direct current interconnections, the use of XLPE cables is a more economical solution compared to the classical paper-oilinsulated ones, due to reduced maintenance and lower production costs. Moreover, HVDC cables also facilitate the connection of networks working at different frequencies or non-synchronized. In case of alternating current interconnections, the use of XLPE cable leads to noticeably lower dielectric losses when compared with oil impregnated paper technology [22].

While the electrical properties of this polymer have been widely studied under AC stress, the behavior of these materials under high DC stress is less well understood and needs thorough investigation. In DC conditions, the electric field distribution is highly dependent on operating conditions (thermal gradient and electric field) and can be affected by electric charges trapped in the insulation. Thus, quantitative evaluation of the state of power cable insulation under DC stress is still a challenge, involving much academic and industrial work. It is believed that new insulating materials must be developed to accommodate the continuous electrical constraint applied to the insulation.

The effect of space charge accumulation in an insulating material can be highly detrimental, causing faults ranging from premature ageing to dielectric breakdown. The measurement of the real distribution of the electric field is therefore of considerable importance for an optimal design of the system's insulation [23]. For polymer-insulated HVDC cables, a major challenge to ensure a good level of reliability is controlling the amount of space charge that accumulates in the dielectric under the effect of electrical stress and thermal gradient.

It is now recognized that, although studies on space charge are absolutely necessary to understand the charge accumulation and relaxation phenomena, direct measurements on power cables (specimen and full size) according to CIGRE specifications are required to quantify the local distortions of the electric field under thermal 
gradient $[24,25]$. Then, long-term insulation behavior under DC stress must be studied to develop adapted insulation lifetime models [26].

\section{IMPACT ON INSULATION SYSTEMS: Operations, Control, AND AsSET MANAGEMENT}

A more active, flexible grid structure introduces new devices and new ways of managing assets. This can impact the lifetime and behavior of insulation systems in various ways. This section highlights areas of research into the impact of smart grid technologies on the operation and lifetime management of insulation systems within assets.

\subsection{CHANGING LOAD PATTERNS}

Perhaps the greatest challenge facing asset managers in the power networks sector is accurately determining the expected remaining life of primary high voltage (HV) assets. The majority of assets will have a design life of 2540 years at the time of installation, based on the anticipated loading conditions and expected maintenance intervals. Depending on the actual loading experienced, the true lifetime can be less (if the load pattern turns out to be more onerous), or considerably more for a benign load pattern. Changing load patterns further underline the need to move from a time based asset management strategy, to one focused on the asset condition [27].

Considerable effort has been expended by the dielectrics community on the development of both offline lifetime models and online condition monitoring techniques which can give an indication of remnant life. Both techniques are expected to advance considerably over the next decade, during which time the load patterns seen at distribution level are likely to change further, due to influences from:

- Greater embedded generation, including from renewables with a more stochastic generation profile

- Increasing penetration of network automation as a means of reducing customer outage durations, with back up route likely to see more switching activity and periods of higher loading

- Participation of consumers and the lower voltages of the distribution network in demand response, which may reduce some peak loadings but simultaneously increase load in other periods

- Greater uptake of electric vehicles (EV), leading to significant growth in overall demand from domestic properties, but at less predictable times

As the loading pattern changes, primary HV assets will experience different thermal, mechanical, and electrical stresses. In turn, this will lead to higher rates of thermal, electrical, mechanical (e.g. excessive wear of the contacts in an OLTC), and chemical ageing (e.g. carbonization and increased water content affecting the oil in OLTCs) [2729]. In many cases, these processes are interlinked (for instance, higher temperatures will accelerate many chemical degradation processes; greater thermal cycling of assets leads to higher mechanical forces being generated).

The development of Smart Grid tools and techniques has attracted considerable research interest worldwide, but evidence of consideration of the effects of their deployment on the condition of the HV assets is not widespread. It is important to note that although many negative effects can be envisioned, positive effects may also exist (for instance, the deployment of demand response techniques may help to reduce peak loadings on some assets). Possibilities exist for smart grid tools to be linked to asset management, with mutual benefits to both network operation and asset maintenance. For example, the work of Hanai et al [30] proposes an optimisation between power flow and maintenance considerations, with a view to finding the best compromise on grounds of both technical performance and cost. This is an area which is underexploited, but does rely heavily upon the development of more accurate lifetime models for the electrical assets.

Of the small number of existing studies, most work which links smart grid deployment to effects on dielectric components considers transformers. The most likely reason for this is that a well-established transformer ageing model exists [31], although it would benefit from greater validation. Factors investigated include changing load patterns due to rising electric vehicle penetration [32], which has demonstrated that smarter charging methods would facilitate greater numbers of vehicles per feeder without the need for network reinforcement. Such methods were also considered to reduce the rate of ageing by limiting the extent of charging during demand peaks. Harmonic content associated with charging is also shown as requiring careful control if excessive transformer ageing is to be prevented [33, 34].

One area of mutual benefit is in demand response reducing peak loads can reduce peak temperatures in distribution transformers, thus reducing the rate of ageing [35]. This is a good example of a case where the Dielectrics and Smart Grid communities can work together to provide maximum possible benefits to the network owners and operators.

\subsection{DYNAMIC RATINGS}

Historically, most HV assets have been selected and subsequently operated using offline, static current rating methods. In many cases, this has led to conservative operation, ensuring that the asset temperatures remain well below limits thus minimizing thermal ageing effects. By contrast, dynamic rating methods use real time measurements of the thermal state of the asset and the surrounding environment (including the rated load allowed on items such as the bushings and OLTC, and the performance of the cooling system) to calculate ratings which better reflect true, short term capability [36]. Given the tight financial controls on network reinforcement and upgrade projects, many utilities are seeking to maximize the utilization of their assets through the use of such techniques 
[37].

From the dielectrics perspective, the key issue is in relation to the acceleration of thermal ageing processes. By routinely operating network assets at higher temperatures, often in a cyclic manner, it seems logical that aging rates will increase. This presents both a challenge and an opportunity for the dielectrics community. Dynamic rating systems produce large amounts of data on asset temperatures, so it may be possible to utilize this data in long term studies on thermal aging under true field conditions.

The DEIS could provide valuable input to the design of future dynamic rating systems. Presently, little or no account is taken of the condition of the asset itself in setting ratings -20 year old assets are treated as equal to new ones of the same design. In the future, the work of the DEIS could lead to asset condition factors being included in dynamic rating models, permitting network operators to optimize the balance between asset utilization now, and long term asset life.

\subsection{INCREASED CONDITION MONITORING}

Electrical insulation systems of $\mathrm{HV}$ assets are continuously subjected to excessive electrical, mechanical and thermal stresses. Moreover, current electricity grids around the world are facing an increasingly aged population of HV assets. The emerging smart grids may also consist of legacy assets. One way to ensure reliable operation of an electricity grid is to continuously monitor the electrical insulation systems for proactive asset management [38].

In the smart grid environment, the prevalence of information and communication technology (ICT) can be utilized for improved condition monitoring and assessment of electrical insulation systems. Figure 1 is a conceptual diagram of the necessary steps for condition monitoring and assessment of electrical insulation systems of in-service assets.

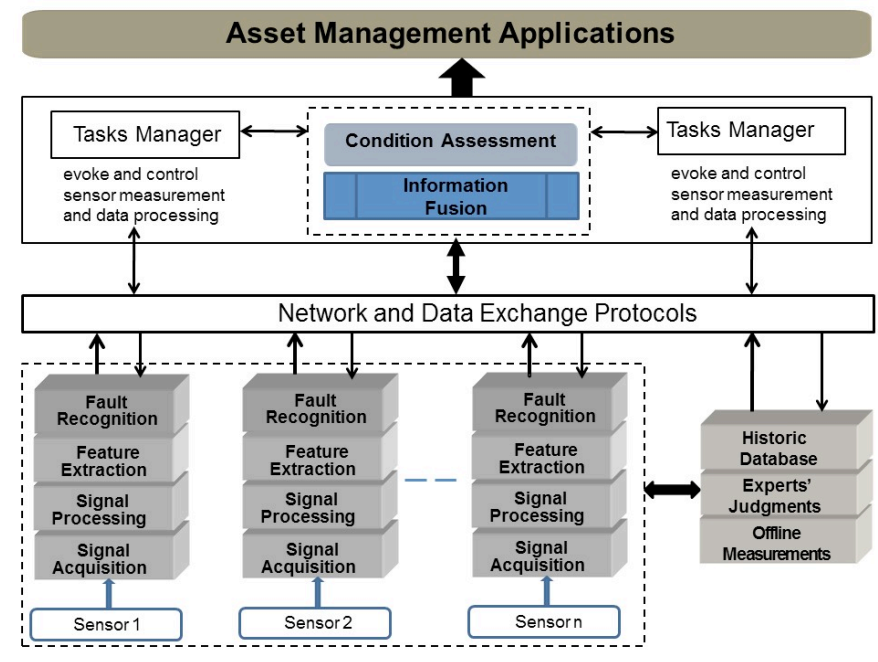

Figure 1: Condition monitoring and assessment of electrical insulation systems of assets in the electricity grid
As shown in Figure 1, a number of challenging issues need to be addressed to develop condition monitoring of insulation systems. They are: (1) efficient deployment of an optimal set of sensors to provide sufficient visibility of the condition of insulation systems; (2) effective signal acquisition and signal processing for extracting data and information from sensor measurements; (3) transformation of data and information into useful knowledge regarding the condition of insulation system (i.e. fault type identification); (4) integration of online sensor measurement and other information (e.g. the original factory test report, the most recent offline measurement, human experts' judgments and onsite inspection) for determining an accurate health status of assets; and (5) intelligent middleware for deploying signal acquisition, signal processing, data mining and information fusion algorithms for monitoring and diagnosis.

To provide dynamic information of system health, a variety of online sensors need to be employed. Examples for a power transformer include temperature, water content in the oil, dissolved gas measurement, leakage current from the test tap of the bushings, and partial discharge (PD) detection and localization. Necessary data acquisition firmware needs to be integrated with the sensors to appropriately acquire and transmit signals for further processing. Sampling rate, frequency range and sensitivity are important factors that can influence the measurements and therefore they need to be carefully selected.

To make an accurate diagnosis, it is necessary to apply digital signal processing techniques to the measured signals to remove the effects of interference and noise in the substation environment. Some widely used de-noising algorithms are the short-time Fourier transform (STFT), notch filter, discrete wavelet transform (DWT), and ensemble empirical mode decomposition (EEMD). An important requirement is that the technique needs to be adaptive and robust, i.e. some parameters of the algorithms can be adjusted automatically according to the characteristics of the measured signals [39].

Interpreting data obtained from online sensor measurements and making explicit condition assessment of insulation systems is non-trivial. Given the potentially large volume of raw data captured, it is necessary to develop an automated procedure to extract useful information and insight. The extracted information must support decisionmaking for operation and maintenance of assets. Feature extraction and pattern recognition techniques can automatically extract representative attributes from raw data, and subsequently identify types of faults occurring in the insulation system. Techniques for feature extraction and pattern recognition are abundant in the literature, and include artificial neural networks (ANNs), evolutionary algorithms, and kernel machines [40].

To provide a full picture of insulation condition, information derived from multiple sensors and sources must be combined or fused together. This includes historic measurement data, offline test reports, and expert judgement, as well as the online information described 
above. Machine learning techniques can be used to construct a model of the links between these sources. Once deployed, the model can be utilized to interpret new measurements and make asset condition assessments [41, 42].

The system shown in Figure 1 is expected to be subject to frequent change, through adding new sensors and algorithms, and updating configurations. Hence, it is desirable to have an intelligent middleware or platform that can be easily reconfigured. Middleware is a suite of software tools which can hide the complexity of the hardware, and facilitate data and information exchange amongst different condition monitoring components [43]. The intelligent middleware allows the deployment of functional entities called agents [44], which can use standards-based communication to exchange data and information for diagnosis and information fusion [45]. Multi-agent systems (built upon agents) can give the capability of handling uncertainties, which is important for practical intelligent condition monitoring and assessment.

\subsection{INCREASED NETWORK DATA}

Traditionally, condition monitoring and asset management were utility functions very distinct from network operation and control. But as shown earlier, the way in which assets are being utilized within the network is changing, and the impact of variable loading, dynamic ratings, and smart grid technologies such as active network management must be considered when assessing insulation system life.

On the one hand, assets are expected to perform closer to their limits than ever before, which increases stress on the insulation and gives the potential for early failure. At the same time, cheaper sensors and ubiquitous computing mean that there is more data about network performance and asset health than ever before. Hence, the tools are available to track external stresses on assets, and give a better understanding of insulation system health.

This would require the integration of various smart grid data analysis systems. Information fusion between systems for condition assessment and, say, power flow management or voltage control would allow asset health to be taken into account when planning control actions, and allow network events such as faults to be considered when assessing insulation health [46]. This type of systems integration requires a platform or method of communication between each sub-system, such as the multi-agent architecture discussed in the previous section. The same issues of upgradability, extensibility, and handling of uncertainty apply [47].

A further implication of the integration of network and condition data is that network effects on insulation systems can be studied to a much greater degree than previously possible. As an example, the effect of harmonics on the phase-resolved PD pattern has been recently documented [48]. While it is possible to infer some details of the harmonic regime from the PD pattern [49], this approach will always be more prone to inaccuracy than direct measurement. The increase in network monitoring and use of phasor measurement units (PMUs) makes it more likely that information about voltage harmonics will be available to online condition monitoring systems. This type of supplementary information will make insulation monitoring and diagnosis more reliable.

\section{MEETING THE SMART GRID CHALLENGE}

The previous sections have surveyed the landscape of smart grid technologies and their impact on insulation systems. Here, consideration is given to the challenge of delivering the promise of the smart grid, and the criticality of dielectrics research to this goal.

At its core, the smart grid aims to be a self-managing, self-healing system with limited human intervention: a much more autonomous version of traditional passive grids. This requires increased monitoring to give greater visibility of the performance and condition of all network components. Monitoring can be used for various ends, including improved condition assessment, dynamic rating, and lifetime estimation of equipment, as discussed earlier. But these ends can only be achieved by comparison of online data with models of expected performance or condition. Therefore, good models of asset behavior are essential to the smart grid vision.

Such models of expected asset behavior can be based on physical understanding (as in [31]) or derived from data (as in [40]), with a physical model considered to be the "gold standard" in terms of accuracy and explainability. However, in many situations it is more feasible to collect quantities of data for empirical modeling, since the understanding of aging and failure processes is simply not advanced enough to create a physical model.

The DEIS is uniquely placed to further the understanding of these processes, and to develop the next generation of materials and asset models required for the self-managing smart grid. There are significant questions about the accuracy of existing models and standards and their transferability from lab conditions to the field. There is also a pressing need for models of aging of new materials and devices, and for materials utilized in new applications such as HVDC. Without furthering the understanding of aging and failure of insulation systems, the type of models required for online condition assessment and dynamic rating cannot be created, and many of the self-managing, self-healing functions of the smart grid will not be fully achieved. As a result, further fundamental research into dielectric phenomena is essential

\section{CONCLUSIONS}

This paper has described a number of smart grid technologies which impact on through-life management of insulation systems. Some have positive benefits, such as new materials with self-healing properties and wider monitoring for improved condition assessment. Some 
require a change to current practices and understanding, such as HVDC and changing load patterns, and therefore come with their downsides as well as advantages. The confluence of new technologies and a desire for change within the industry means that grids will continue to evolve to meet the needs of society. As a community, we must be aware of the impacts of new technologies on traditional assets, and the opportunities these same technologies present for improving the operation and maintenance of $\mathrm{HV}$ assets. Through on-going fundamental research into dielectric materials, failure modes, and test techniques for insulation systems, the DEIS has a cornerstone role to play in the future of smart grids.

\section{REFERENCES}

[1] SMB Smart Grid Strategic Group (SG3), "IEC Smart Grid Standardization Roadmap," 2010.

[2] EPRI, "Smart Grid Roadmap and Architecture," 2010.

[3] International Energy Agency, "Smart Grids Technology Roadmap," 2011.

[4] National Institute of Standards, "NIST Framework and Roadmap for Smart Grid Interoperability Standards," 2010.

[5] European Technology Platform, "Strategic Research Agenda for Europe's Electricity Networks of the Future," 2012.

[6] State Grid Corporation of China, "SGCC Framework and Roadmap for Strong and Smart Grid Standards," 2012.

[7] R. Frei, R. McWilliam, B. Derrick, A. Purvis, A. Tiwari, and G. D. M. Serugendo, "Self-healing and self-repairing technologies," International Journal of Advanced Manufacturing Technology vol. 69, pp. 1033-1061, 2013.

[8] D. A. Hoch, J. P. Reynders, and R. E. Macey, "A Silicone Based Hydrophobic Coating for High Voltage Insulators " presented at the 3rd AFRICON Conference, 1992.

[9] J. Rocks, U. Kaltenbom, and T. Hucke, "Intrinsic Hydrophobic Epoxies for Outdoor Insulation," presented at the Conference on Electrical Insulation and Dielectric Phenomena (CEIDP), 2000.

[10] P. K. Dubey, A. Dixit, and P. Kamphiranon, "Self Healing Epoxy System for Outdoor Electrical Applications," presented at the Annual Meeting Thermoset Resin Formulators Association, 2013.

[11] B. S. Bernstein, "Assessment of Self-Healing Polymer Technology for Utility Overhead Applications," IEEE Electrical Insulation Magazine, vol. 22, pp. 15-20, 2006.

[12] Z. W. Lu, H. Janssen, A. Herden, and H. C. Kärner, "Generation of LMW Components in Silicone Rubbers," presented at the IEEE Conference on Electrical Insulation and Dielectric Phenomena (CEIDP), Austin, Texas, 1999.

[13] G. G. Karady, M. Shah, and R. L. Brown, "Flashover mechanism of silicon rubber insulators used for outdoor insulation part 1," IEEE Transactions on Power Delivery, vol. 10, pp. 1965-1971, 1995.

[14] Y. Zhou, F. Wang, C. Li, H. Jiang, and H. Cui, "Characteristic of Field-Aged $500 \mathrm{kV}$ Composite Insulators," presented at the Conference on Electrical Insulation and Dielectric Phenomena (CEIDP), 2011.

[15] J. Mackevich and M. Shah, "Polymer Outdoor Insulating Materials Part 1: Comparison of Porcelain and Polymer Electrical Insulation," IEEE Electrical Insulation Magazine, vol. 13, pp. 5-12, 1997.

[16] V. K. Agarwal, "Aging of Multi-Stressed Polymeric Insulators," IEEE Transactions on Electrical Insulation, vol. 24, pp. 741-764, 1989.

[17] S. Wei, Z. Linjie, and L. Chengrong, "On-line detection methods of composite insulator," High Voltage Engineering, Wuhan, China, vol. 31, pp. 28-30, 2005.

[18] B. Li, X. Wang, and L. Nian, "Remote Online Monitoring System For Suspension Insulator Strings," presented at the IEEE International Symposium on Industrial Electronics, 2006.

[19] J. Wang, C. Yao, Y. Mi, X. Zhang, and C. Li, "Research for the LED optical fiber sensor for the leakage current of the insulator string," presented at the Asia-Pacific Power and Energy Engineering Conference (APPEEC), 2012.
[20] S. C. Oliveira and E. Fontana, "Optical System for Flashover Prediction In High Voltage Transmission Lines," presented at the International Microwave and Optoelectronics Conference (IMOC 2007), 2007.

[21] H. Li, X. Wen, N. Shu, and C. Pei, "Application of Acoustic Emission Technology on Automation Detection of Polluted Insulator," presented at the International Conference on Mechatronics and Automation (ICMA 2009), 2009.

[22] C. R. Bayliss and B. J. Hardy, Transmission and Distribution Electrical Engineering: Elsevier, 2012.

[23] J. Castellon, P. Notingher Jr, S. Agnel, A. Toureille, J. F. Brame, P. Mirebeau, et al., "Electric field and space charge measurements in thick power cable insulation under long term ac and dc stress using the thermal step method," IEEE Electrical Insulation Magazine, vol. 25, pp. 30-42, 2009.

[24] M. Fu, F. Perrot, C. Green, S. Dodd, J. Fothergill, J. Castellon, et al., "HVDC cables during a long-term ageing program," presented at the INSUCON, Birmingham, 2013.

[25] Cigre SC B1, "Recommendations for Testing DC Extruded Cable Systems for Power Transmission at a Rated Voltage up to $500 \mathrm{kV}$, Brochure 496," ed, 2012.

[26] G. Mazzanti, G. C. Montanari, and L. A. Dissado, "Electrical ageing and life models: The role of space charge," IEEE Transactions on Dielectrics and Electrical Insulation vol. 12, pp. 876-890, 2005.

[27] S. Bahadoorsingh and S. Rowland, "A Framework Linking Knowledge of Insulation Aging to Asset Management," IEEE Electrical Insulation Magazine, vol. 24, pp. 38-46, 2008.

[28] J. Densley, "Ageing mechanisms and diagnostics for power cables an overview," IEEE Electrical Insulation Magazine, vol. 17, pp. 1422, 2001.

[29] M. Ali, C. Eley, A. Emsley, R. Heywood, and X. Xaio, "Measuring and understanding the ageing of kraft insulating paper in power transformers," IEEE Electrical Insulation Magazine, vol. 12, pp. 2834, 1996.

[30] M. Hanai, H. Kojima, N. Hayakawa, K. Shinoda, and H. Okubo, "Integration of Asset Management and Smart Grid with Intelligent Grid Management System," IEEE Transactions on Dielectrics and Electrical Insulation, vol. 20, pp. 2195-2202, 2013.

[31] IEC, "IEC 60076-7: Power Transformers: Part 7: Loading guide for oil-immersed power transformers," ed, 2005.

[32] A. Hilshey, P. Hines, P. Rezai, and J. Dowds, "Estimating the Impact of Electric Vehicle Smart Charging on Distribution Transformer Aging," IEEE Transactions on Smart Grid, vol. 4, pp. 905-913, 2013.

[33] R. Ghunem, M. Hamid, S. Jayaram, R. Seethapathy, and A. Naderian, "Transformer Insulation Risk Assessment under Smart Grid Environment due to Enhanced Aging Effects," presented at the IEEE Electrical Insulation Conference, Annapolis, 2011.

[34] M. Masoum, P. S. Moses, and S. Deilami, "Load Management in Smart Grids Considering Harmonic Distortion and Transformer Derating," presented at the Innovative Smart Grid Technologies, Gaithersburg, 2010.

[35] J. Jargstorf, K. Vanthournout, T. D. Rybel, and D. Van Hertem, "Effect of Demand Response on Transformer Lifetime Expectation," presented at the 3rd IEEE PES Innovative Smart Grid Technologies Europe, Berlin, 2012.

[36] G. J. Anders, A. Napieralski, M. Zubert, and M. Orlikowski, "Advanced modeling techniques for dynamic feeder rating systems," IEEE Transactions on Industry Applications, vol. 39, pp. 619-626, 2003.

[37] D. A. Douglass, D. C. Lawry, A.-A. Edris, and E. C. Bascom, "Dynamic Thermal Ratings Realize Circuit Load Limits," IEEE Computer Applications in Power, vol. 13, pp. 38-44, 2000.

[38] T. K. Saha, "Review of Modern Diagnostic Techniques for Assessing Insulation Condition in Aged Transformers," IEEE Transactions on Dielectrics and Electrical Insulation, vol. 10, 2003.

[39] J. C. Chan, H. Ma, T. K. Saha, and C. Ekanayake, "Self-Adaptive Partial Discharge Signal Extraction Based on Ensemble Empirical Mode Decomposition and Optimal Morphological Thresholding," IEEE Transactions on Dielectrics and Electrical Insulation, vol. 21, 2014.

[40] H. Ma, J. C. Chan, T. K. Saha, and E. Ekanayake, "Pattern Recognition Techniques and their Applications for Automatic Partial 
Discharge Source Classification," IEEE Transactions on Dielectrics and Electrical Insulation, vol. 20, 2013.

[41] H. Ma, T. K. Saha, and C. Ekanayake, "Statistical Learning Techniques and their Applications for Condition Assessment of Power Transformers," IEEE Transactions on Dielectrics and Electrical Insulation, vol. 19, 2012.

[42] V. M. Catterson, S. D. J. McArthur, and G. Moss, "Online Conditional Anomaly Detection in Multivariate Data for Transformer Monitoring," IEEE Transactions on Power Delivery, vol. 25, pp. 2556-2564, 2010.

[43] N. Cai, M. Gholami, L. Yang, and R. W. Brennan, "ApplicationOriented Intelligent Middleware for Distributed Sensing and Control," IEEE Transactions on Systems, Man, and Cybernetics Part C: Applications and Reviews, vol. 42, 2012.

[44] S. D. J. McArthur, E. M. Davidson, V. M. Catterson, A. L. Dimeas, N. D. Hatziargyriou, F. Ponci, et al., "Multi-Agent Systems for Power Engineering Applications-Part 1: Concepts, Approaches, and Technical Challenges," IEEE Transactions on Power Systems, vol. 22, pp. 1743-1752, 2007.

[45] S. D. J. McArthur, E. M. Davidson, V. M. Catterson, A. L. Dimeas, N. D. Hatziargyriou, F. Ponci, et al., "Multi-Agent Systems for Power Engineering Applications-Part 2: Technologies, Standards, and Tools for Building Multi-Agent Systems," IEEE Transactions on Power Systems, vol. 22, pp. 1753-1759, 2007.

[46] V. M. Catterson, E. M. Davidson, and S. D. J. McArthur, "Embedded Intelligence for Electrical Network Operation and Control," IEEE Intelligent Systems, vol. 26, pp. 38-45, 2011.

[47] E. M. Davidson, V. M. Catterson, and S. D. J. McArthur, "The role of intelligent systems in delivering the Smart Grid," presented at the IEEE Power and Energy Society General Meeting, 2010.

[48] M. Florkowski, B. Florkowski, J. Furga, and P. Zydron, "Impact of High Voltage Harmonics on Interpretation of Partial Discharge Patterns," IEEE Transactions on Dielectrics and Electrical Insulation, vol. 20, pp. 2009-2016, 2013.

[49] V. M. Catterson, S. Bahadoorsingh, S. Rudd, S. D. J. McArthur, and S. M. Rowland, "Identifying Harmonic Attributes from Online Partial Discharge Data," IEEE Transactions on Power Delivery, vol. 26, pp. 1811-1819, 2011 